\title{
Heart failure on peritoneal dialysis: Where do we stand?
}

\author{
Marina Reis $^{1}$ iD, Catarina Almeida ${ }^{1}$ (D) , Ana Marta Gomes ${ }^{1}$ iD, João Carlos Fernandes ${ }^{1}$ iD \\ ${ }^{1}$ Nephrology Department, Centro Hospitalar Vila Nova de Gaia/Espinho, Vila Nova de Gaia, Portugal
}

\section{ABSTRACT}

Cardiovascular disease continues to be the most frequent cause of death in peritoneal dialysis patients and an important obstacle for the improvement of technique survival. Heart failure diagnosis and management is particularly challenging among dialysis patients, and this condition remains underdiagnosed and undertreated in this population. The most common phenotype of heart failure among peritoneal dialysis patients is heart failure with preserved ejection fraction, diastolic disfunction and left ventricular hypertrophy. Unfortunately, unlike what happens with heart failure with reduced ejection fraction, there is lack of evidence to support a specific drug regimen to treat heart failure with preserved ejection fraction. Several conditions associated with end stage kidney disease, such as anemia, hyperphosphatemia, secondary hyperparathyroidism, inflammation, and insulin resistance seem to be involved in the pathogenesis of heart failure with preserved ejection fraction and for this reason, the term uremic cardiomyopathy has been proposed. There is a lack of evidence regarding the optimal heart failure treatment for peritoneal dialysis patients and more studies are needed to assess the efficacy and safety of the new drugs available for heart failure treatment. This review explores the spectrum of heart failure on peritoneal dialysis, its pathogenesis, risk factors and possible therapeutic and preventive measures.

Keywords: heart failure, cardiovascular disease, peritoneal dialysis

(C) 2021 Portuguese Journal of Nephrology \& Hypertension. Published by Publicações Ciência \& Vida This is an open access article under the CC BY-NC-ND license (http://creativecommons.org/licenses/by-nc-nd/4.0/).

\section{INTRODUCTION}

Cardiovascular disease (CVD) continues to be the most frequent cause of death in dialysis patients. ${ }^{1-3}$ Whichever the dialysis modality, heart failure (HF) and CVD need an improved approach that is currently limited by the few studies on this population. In Portugal, CVD was responsible for $41.5 \%$ of PD deaths in $2019 .{ }^{3}$ CVD manifests itself as acute myocardial infarction, cerebrovascular events, arrhythmia, HF and sudden cardiac death. HF especially represents a high burden for PD patients since it is responsible for a high number of hospitalizations. In a 4-year follow-up study with 220 PD patients, $28 \%$ developed new-onset HF and $62 \%$ of those with previous HF had an episode of worsening HF requiring hospitalization. The cumulative 4-year survival probability was $37.4 \%$ and $64.7 \%$ for patients with and without previous HF, respectively. ${ }^{4}$ The major phenotype of HF on PD is diastolic dysfunction and marked left ventricular hypertrophy (LVH). ${ }^{4-6}$ Risk factors for HF in these patients include those that affect the general population as well as those related to end-stage kidney disease (ESKD) and those that are specific to PD. ${ }^{7-10}$ PD has also some potential cardiovascular advantages, in particular the avoidance of the unphysiological fluctuations in fluid and solute status associated with hemodialysis. Unquestionably, the longevity of patients on PD is related to improvements in the diagnosis, management, and prevention of CVD and HF, but little is known about the physiopathology of both, on PD patients. In this review, we will explore the spectrum of HF on PD patients, its pathogenesis, risk factors and possible therapeutic and preventive measures.

\section{HEART FAILURE ON PERITONEAL DIALYSIS}

HF diagnosis is challenging in PD patients. Echocardiography, the most used method for left ventricle evaluation, is volume-dependent and parameters such as elevated left ventricular end-diastolic pressure, increased estimated pulmonary artery systolic pressure or left ventricular ejection fraction may be altered on patients with hypervolemia. ${ }^{11}$ International Society for Peritoneal Dialysis (ISPD) guidelines suggest an echocardiography evaluation after PD initiation for assessment of LVH and systolic and diastolic function as well as cardiac valvular abnormalities including valvular calcification. ${ }^{12} \mathrm{~A}$ reassessment should be carried out whenever clinical status change. Likewise, $\mathrm{N}$ terminal pro-brain natriuretic peptide (NT-proBNP) is an important $\mathrm{HF}$ biomarker and its levels have been found to be higher in patients on dialysis than in the general population. ${ }^{13,14}$ NT-proBNP elevation in PD patients is an important predictor of cardiovascular congestion, mortality, and adverse cardiovascular outcomes and seems to add important prognostic information beyond that provided by LVH, systolic dysfunction, and other conventional risk factors. ${ }^{13,14}$

Based on left ventricle function, $\mathrm{HF}$ is classified into two major types with different physiopathology and treatment options: heart 
failure with preserved ejection fraction (HFpEF) and heart failure with reduced ejection fraction (HFrEF). ${ }^{15}$ The main phenotype on PD patients is HFpEF with diastolic dysfunction and marked LVH, also known as uremic cardiomyopathy. ${ }^{4-6}$

\section{Heart failure with preserved ejection fraction - Uremic cardiomyopathy}

Almost $70 \%$ of patients starting PD have detectable LVH by echocardiography and changes in cardiac structure tend to progress during dialysis treatment. ${ }^{16} \mathrm{LVH}$ is the strongest independent predictor of cardiovascular mortality in patients with ESKD independently of other risk factors. ${ }^{10,16-18}$ Diastolic dysfunction seems to be related to a decrease in the active reuptake of calcium into the sarcoplasmic reticulum as well as to ventricular fibrosis, which impairs passive relaxation and precedes $\mathrm{LVH}^{6,19}$ The pathogenesis of uremic cardiomyopathy on dialysis patients seems to be multifactorial, and available evidence strongly suggests that inflammation, FGF23 and low Klotho levels affect cardiac remodeling and therefore are involved in the development of diastolic disfunction and LVH. ${ }^{6,20}$ Early diastolic disfunction is difficult to diagnosis but since it is high prevalent on PD patients, other echocardiographic techniques such as tissue doppler velocities should be considered in order to identify myocardial dysfunction at the preclinical stage and implement interventions to prevent its progression. ${ }^{21}$

\section{Heart failure with reduced ejection fraction}

ISPD guidelines recommend that PD patients who have a persistent reduction in ejection fraction of less than 40 percent should be further evaluated for coronary heart disease (CHD). ${ }^{12}$ The prevalence of $\mathrm{CHD}$ is much higher than that observed in the general population but may still be underdiagnosed since CHD often has an atypical presentation in PD patients. ${ }^{22}$ Also, clinically silent CHD is common among dialysis patients and conveys a similar negative prognosis compared with a clinically recognized myocardial infarction. ${ }^{22,23}$ Its diagnosis remains challenging since noninvasive methods used for diagnosis of silent CHD in the general population such as troponin levels, electrocardiography, stress echocardiography and pharmacological perfusion imaging, lack accuracy for CHD surveillance among PD patients. ${ }^{12,22,23}$

\section{RISK FACTORS FOR HEART FAILURE AND CARDIOVASCULAR DISEASE ON PERITONEAL DIALYSIS PATIENTS}

\section{General risk factors}

PD patients share some risk factors for $\mathrm{HF}$ and CVD with the general population, such as age or male sex. ${ }^{8}$ (Table I) However, the effect of obesity on cardiovascular risk on PD patients is inconsistent. Some studies have found that obesity confers a survival advantage, 24,25 whereas others have reported an association of obesity with an increased risk of mortality, ${ }^{26}$ or no association at all. ${ }^{8}$ Dyslipidemia is an established risk factor for CVD in the general population, but data is limited with regards to PD. In theory, PD provides an ideal milieu for worsening dyslipidemia, both by continuous glucose absorption and significant albumin loss, although evidence of such cause-and-effect link is sparse. ${ }^{27}$ Current guidelines do not recommend starting statins on patients already on dialysis, although a recent study on hemodialysis patients showed that statin therapy, preferably combined with ezetimibe, was associated with a lower risk of all-cause mortality. ${ }^{27,28}$ Glycemic control, which is also an established risk factor for CVD in diabetics, is difficult in PD patients, due to the constant glucose absorption. 2,7,9,29 In addition to glycemic control in diabetic patients, studies suggest that PD patients with morning blood sugar values between 100 and $126 \mathrm{mg} / \mathrm{dl}$ are at a higher risk of death compared to those with levels below $100 \mathrm{mg} / \mathrm{dl}^{2}{ }^{2}$ Therefore, periodic glycemic control is important even in non-diabetic PD patients, and in individuals in whom glycemic values are elevated and insulin resistance is suspected, aggressive lifestyle interventions should be implemented.

\section{Risk factors related to end-stage kidney disease}

Several potential factors have been investigated to explain the high prevalence of endothelial dysfunction and cardiovascular mortality among PD and hemodialysis patients(Table II). Hyperphosphatemia, hyperparathyroidism, and hypercalcemia (caused by high doses of vitamin D or calcium-based phosphate binders) have been implicated in the calcification process which is a significant risk factor for hypertension, cardiac remodeling, LVH and cardiovascular mortality in ESKD. ${ }^{2,6,9}$ Even without atherosclerosis, coronary medial calcification

\section{Table I}

Risk factors for heart failure and cardiovascular disease in dialysis patients.

\begin{tabular}{c|c|c|c|}
\hline General Population & Related to ESKD & Related to PD & Related to HD \\
\hline Age & Anemia & Loss of RRF \\
Male sex & CKD-MBD & Hypertension \\
Smoking & Inflammation & Hypervolemia \\
Dyslipidemia & Protein-energy wasting syndrome & Peritonitis \\
Diabetes Mellitus & Hyperhomocisteinemia & Myocardial stunning \\
& Vitamin D deficiency & Hypertension \\
& Oxidative stress & Loss of RRF \\
& Uremic toxins & \\
\hline
\end{tabular}

AVF - arteriovenous fistula; ESKD - end-stage kidney disease; CKD-MBD - chronic kidney disease-mineral bone disease; HD - hemodialysis; PD - peritoneal dialysis; RRF - renal residual function 


\section{Table II}

Interventions for improvement of cardiovascular and metabolic control of peritoneal dialysis patients

General

\begin{tabular}{l} 
Physical activity; \\
Salt restriction (<2g sodium or $5 \mathrm{~g}$ sodium chloride per day); \\
Stop smoking and reduction of alcohol intake; \\
\hline \multicolumn{1}{c}{ Preserving RRF } \\
\hline Consider an ACEI/ARB as tolerated; \\
Avoid nephrotoxic drugs; \\
Use of biocompatible solutions; \\
\hline
\end{tabular}

Optimize diuretics on patients with RRF and dialysis prescription to achieve euvolemia; Avoid hypotension and dehydration;

Consider use of multifrequency bioimpedance to increase the accuracy of the diagnosis of volume overload;

Diabetes Mellitus

HbA1c should be targeted around $7 \%$ or $8.5 \%$ in older patients;

Icodextrin should be considered as the long-dwell dialysis solution in diabetic

peritoneal dialysis patients for better ratio of ultrafiltration $\mathrm{mL} / \mathrm{g}$ of glucose, and

possibly also as a tool for improving glycemic control;

Inflammation;

Persistently elevated CRP should be investigated for any treatable cause of inflammation;

\begin{tabular}{c}
\hline Nutritional status \\
\hline Dietary protein intake of $1.2-1.3 \mathrm{~g} / \mathrm{kg}$ body weight/day; \\
\hline Blood Pressure \\
\hline Maintain blood pressure $<140 \mathrm{mmHg}$ systolic and $<90 \mathrm{mmHg}$ diastolic; \\
\hline Dyslipidemia \\
\hline
\end{tabular}

Starting statins or statin/ezetimibe combination on patients already on peritoneal dialysis patients is debatable;

In patients already receiving statins or statin/ezetimibe combination at the time of dialysis initiation, these agents can be continued.

ACEI-angiotensin-converting enzyme inhibitor; ARB - angiotensin-receptor blocker; CRP - C Reactive Protein; RRF - residual renal function

may cause decreased diastolic filling, and increased ventricular afterload. ${ }^{30}$ Klotho seems to prevent the development of LVH via blocking the activation of the renin-angiotensin-aldosterone system (RAAS), the sympathetic nervous system and transforming growth factor- $\beta$ and could be a potential therapeutic target. 2,6

Evidence suggests that 25-hydroxyvitamin D levels are lower in PD patients, but whether supplementation could prevent cardiovascular events is not yet proved. Wang et al. confirmed that low serum 25-hydroxyvitamin D levels were associated with an increased risk of fatal or nonfatal cardiovascular events, but in that study the influence of vitamin D on cardiovascular outcomes seemed to closely relate to residual renal function (RRF), severity of $\mathrm{LVH}$, and cardiac dysfunction. ${ }^{31}$

Anemia is a significant predictor of death in patients undergoing PD. ${ }^{32}$ Potential mechanisms that may explain the relationship between anemia and the development of LVH include reduced oxygen delivery to the myocardium, increased cardiac output, increased oxidative stress and activation of the sympathetic nervous system. ${ }^{4,7}$ Also, erythropoietin may have direct effects on myocardial function, since the treatment of severe anemia with erythropoietin stimulating agents is associated with improvement of $\mathrm{LVH} .^{33}$

Chronic inflammation, as demonstrated by an elevated $\mathrm{C}$ reactive protein (CRP), is associated with higher cardiovascular risk in PD patients. ${ }^{4,7}$ Since malnutrition, inflammation, and atherosclerotic CVD often coexist, these risk factors have been proposed to be pathophysiologically linked and are referred to as the MIA syndrome. ${ }^{34}$

\section{RISK FACTORS RELATED TO PERITONEAL DIALYSIS}

There are inherent factors in the PD technique that increase the risk of CVD. Comparison between continuous ambulatory peritoneal dialysis (CAPD) and automated peritoneal dialysis (APD) have been made in terms of cardiovascular risk, but the variability of prescriptions among studies makes impossible to draw conclusions. ${ }^{35,36}$ Therefore, based on current data, no modality should be preferred to the other based on the cardiovascular risk of the patient.

\section{Dialysis Solution}

The continuous exposure to dialysate glucose leads to the formation of advanced glycation end-products that are associated with peritoneal membrane damage and loss of RRF. ${ }^{7}$ Advanced glycation end-products could also have a role in the cardiovascular risk of PD patients, but randomized studies with both biocompatible and standard solutions have not shown better survival outcomes. ${ }^{37}$ Several studies have suggested clinical benefits of icodextrin solutions regarding fluid management and improved glycemic and metabolic control. $^{38,39}$ It is also hypothesized, that the instillation of the dialysis solution into the peritoneal cavity could result in an elevation of systemic blood pressure due to a rise in total peripheral resistance even in the absence of hypervolemia. ${ }^{7}$

\section{Loss of residual renal function}

Loss of RRF is an important risk factor for all-cause mortality and CVD among PD patients. ${ }^{7-9,40,41}$ The CANUSA study revealed that each $5 \mathrm{~L}$ per week per $1.73 \mathrm{~m}^{2}$ increment corresponded to a $12 \%$ decrease in the relative risk of death. ${ }^{40}$ The ADEMEX study also corroborates this data. ${ }^{41}$ More recently, a prospective study by Wang et al. in 246 CAPD patients, $39 \%$ of which were completely anuric, demonstrated more adverse cardiovascular, inflammatory, nutritional, and metabolic profiles and a higher overall mortality in the anuric population when compared to those patients with a residual glomerular filtration rate superior to $1 \mathrm{~mL} / \mathrm{min} / 1.73 \mathrm{~m}^{2} .42$

\section{Hypervolemia and hypertension}

Hypervolemia is common in PD patients and is associated with development of endothelial disfunction, $\mathrm{LVH}$ and consequently poor outcomes. ${ }^{7-9}$ Since hypertension is a consequence of hypervolemia 
in most cases, targeting an adequate dry weight is also essential for blood pressure control. Hypertension is as well directly correlated with LVH and diastolic dysfunction and consequently an important risk factor for HF. ${ }^{7-9,43}$ Hypervolemia can occur due to loss of RRF, high sodium and water intake or due to ultrafiltration failure. PD prescriptions should also be tailored to optimize ultrafiltration and volemia. This includes, for example, the adjustment of the dwell time to the peritoneal transport status and the use of icodextrin for the long dwell. ${ }^{12}$ Various studies validate the impact of hypervolemia on cardiac function, highlighting the importance of a close monitorization of volume status of PD patients to prevent HF progression. ${ }^{8-10,44}$

Multifrequency bioimpedance can be a useful tool to increase the accuracy of the diagnosis of volume overload. Despite its usefulness, bioimpedance still does not discriminate the amount of extracellular water that is in the intravascular compartment, available for ultrafiltration, and which is in the interstitial compartment and that should not be removed at a higher rate. ${ }^{45} \mathrm{HF}$ patients have a narrow safety window for euvolemia, and a stepwise weight reduction is crucial to avoid end-organ ischemia.

\section{Peritonitis}

Episodes of peritonitis have been shown to correlate with increased mortality. In a study of 1321 PD patients, peritonitis was independently associated with increased risks of all-cause, cardiovascular, and infection-related mortality. ${ }^{46}$ Furthermore, it has been shown that severe or repeated peritonitis may be associated with deterioration of peritoneal membrane status and UF capacity, leading to worse volume control. ${ }^{7}$ Also, a chronic inflammatory state may persist even after successful treatment of peritonitis which may predispose to enhanced cardiovascular risk. ${ }^{9}$

\section{INTERVENTIONS FOR PREVENTION AND TREATMENT OF HEART FAILURE ON PD PATIENTS}

Since CVD and particularly HF is responsible for high mortality and morbidity, it is crucial to implement measures to prevent the progression of HF on PD patients. Table II shows measures recommended by ISPD, KDIGO and ERBP guidelines for a better cardiovascular and metabolic control. $12,27,47$

HF treatment in PD patients is not well defined due to the shortage of studies in this population. Selected cases could be candidates for implantable cardioverter defibrillator (ICD) or nodal auricular ablation for atrial fibrillation. ${ }^{15,48}$ Some data report a higher rate of peri-procedure complications in dialysis patients. Furthermore, dialysis patients have historically been excluded from ICD trials, as the competing risks associated with renal failure were anticipated to diminish the benefits of device therapy. ${ }^{48}$ There are no guidelines for these procedures on dialysis patients, so decisions must be made on a case-by-case basis. In line with European Society of Cardiology (ESC) guidelines, $\mathrm{HF}$ treatment is different in $\mathrm{HFpEF}$ and $\mathrm{HFrEF}$ and is therefore presented in separate topics in this review. ${ }^{15}$

\section{Treatment of heart failure with preserved ejection fraction /uremic cardiomiopathy}

An important component of treating a patient with HFpEF is to treat the concomitant cardiovascular conditions, such as hypertension, CHD, atrial fibrillation, and non-cardiovascular diseases such as chronic pulmonary disease, anemia, diabetes mellitus and sleep disorders. Compared with HFrEF patients, hospitalizations and deaths in patients with HFpEF are more likely to be non-cardiovascular. ${ }^{15}$ Trials of angiotensin converting enzyme inhibitors (ACEIs), angiotensin receptor blockers (ARBs), beta-blockers and mineral receptor antagonists (MRAs) have all failed to reduce mortality in patients with HFpEF in the general population and there is a lack of evidence to support a specific drug regimen to treat HFpEF in the PD population. ${ }^{12,15}$ As explained above, evidence suggests that treating hypertension could be important for preventing HFpEF progression. Hypertension treatment involves recognition and control of volume overload, lowering dietary sodium intake, appropriate use of loop diuretics in the presence of substantial RRF, adaptation of dialysis regimen to peritoneal transporting status, and use of icodextrin solutions during long daytime dwell in APD or during the long overnight dwell in CAPD. Antihypertensive drug therapy should only be initiated when hypertension remains uncontrolled despite the adequate management of volume. Although comparative efficacy and safety of different antihypertensive drug categories among patients on PD remains elusive, some clinical studies suggest using ACEIs/ARBs as first-line agents. ${ }^{12}$ Further, RAAS blockers are associated with slower decline in RRF and also with peritoneal membrane function preservation. ${ }^{49}$

According to the ISPD guidelines, in patients with LVH or dilated cardiomyopathy, treatment with a beta-blocker should be considered. ${ }^{12}$ In hemodialysis patients, its use improved left ventricular remodeling and functional class in patients with dilated cardiomyopathy sustained for a period of 24 months and showed significant benefit in cardiovascular mortality, and HF related and all-cause hospitalizations. ${ }^{50}$ Studies evaluating the influence of beta-blockers in $\mathrm{LVH}$ and diastolic disfunction in PD patients are lacking.

In patients already receiving ACEIs/ARBs treatment with MRAs should be considered. Spironolactone has been shown to significantly reduce the progression of left ventricular mass index over a 24-month period in PD patients compared with controls. There was no significant difference in the occurrence of hyperkalemia between the two groups. $^{51}$

As stated above, anemia is a risk factor for LVH and HF. There is insufficient data to recommend a different approach and $\mathrm{Hb}$ goals in patients with or without $\mathrm{HF}$, so the recommendation for anemia treatment in PD patients should follow KDIGO 2012 recommendations. ${ }^{12,47}$ Iron deficiency is associated with a worse prognosis in HF patients. Intravenous iron supplementation has been studied in patients with $\mathrm{HF}$ and iron deficiency (serum ferritin $<100 \mathrm{mg} / \mathrm{L}$ or ferritin between 100 and $299 \mathrm{mg} / \mathrm{L}$ and transferrin saturation <20\%) both with and without anemia. In the FAIR-HF trial, intravenous ferric carboxymaltose has been shown to improve self-reported patient global assessment and NYHA class. ${ }^{52}$ Although these studies were not realized in PD patients, it seems reasonable to give special attention to iron metabolism in patients with HF even without anemia. 
The correction of abnormalities on chronic kidney disease - mineral bone disease (CKD-MBD) metabolism might have beneficial effects on LVH. In patients with secondary hyperparathyroidism on hemodialysis, intravenous calcitriol induced regression in myocardial hypertrophy and improved cardiac systolic and diastolic function. ${ }^{53}$ Results with cinacalcet are contradictory. The EVOLVE trial, which included 2,602 patients with secondary hyperparathyroidism on hemodialysis, reported that treatment with cinacalcet seemed to have beneficial effects on cardiovascular end points in older patients. ${ }^{54} \mathrm{~A}$ posterior meta-analysis reported that treatment with cinacalcet was not associated with a beneficial effect on cardiovascular outcomes, such as LVH, CHD or cardiac function. ${ }^{55}$ Supplementation with cholecalciferol could be considered. ${ }^{30}$

Restoration and maintenance of sinus rhythm is preferred when atrial fibrillation occurs in patients with HFpFE. When this cannot be achieved, rate control becomes essential, preferably with a cardio selective beta-blocker. Correction of valvular disease should also be considered in appropriate patients. ${ }^{12,15}$

Novel therapies targeting inflammation, oxidative stress, and uremic toxins could be the next step for uremic cardiomyopathy treatment. ${ }^{6}$ A few studies have investigated the effect of antioxidants such as Vitamins C, E, statins, omega-3 fatty acids, and N-acetylcysteine. Oral administration of $\mathrm{N}$-acetylcysteine was the most encouraging and was associated with suppression of oxidative stress, inhibition of local and systemic inflammation, prevention of loss of RRF and prevention of peritoneal membrane dysfunction. ${ }^{56,57}$

\section{Treatment of heart failure with reduced ejection fraction}

Treatment options for HFrEF are better established (Figure 1). According to ESC guidelines, ACEI should be considered in patients with stable CHD to prevent or delay the onset of HF. Hydralazine and isosorbide dinitrate may be considered in symptomatic patients with HFrEF who cannot tolerate neither an ACEI nor an ARB to reduce the risk of death. Beta-blockers are recommended in patients with a history of myocardial infarction, to prevent or delay the onset of HF. ${ }^{15}$

Concerning the treatment with MRA on PD patients with HFrEF, spironolactone improved left ventricular ejection fraction without a significant increase in potassium levels. ${ }^{51}$

According to ESC guidelines, ivabradine should be considered to reduce the risk of $\mathrm{HF}$ hospitalization or cardiovascular death in symptomatic patients with ejection fraction $\leq 35 \%$, in sinus rhythm and a resting heart rate over or equal to $70 \mathrm{bpm}$ despite treatment with an evidence-based dose of beta-blocker, ACEI/ARB and an MRA. ${ }^{15}$ At present, no data for patients with glomerular filtration rate inferior to $15 \mathrm{~mL} / \mathrm{min} / 1.73 \mathrm{~m}^{2}$ is available. Although efficacy and safety may be preserved in these patients too, more trials are needed, and ivabradine should be used carefully or avoided in ESKD patients. ${ }^{58}$

A new therapeutic class of agents acting on the RAAS and the neutral endopeptidase system has been developed, the angiotensin receptor neprilysin inhibitor (ARNI). By inhibiting neprilysin, the degradation of natriuretic peptides and bradykinin is slowed. Elevation of circulating A-type natriuretic peptide (ANP) and NT-proBNP is a compensatory mechanism that leads to vasodilation, natriuresis and diuresis, lowers blood pressure, lowers sympathetic tone, and reduces aldosterone levels. The natriuretic peptide system works antagonistically to the RAAS and has favorable effects on the pathogenesis of $\mathrm{HF}$, enhancing myocardial relaxation and preventing remodeling. ${ }^{59}$ The association of sacubitril, an ARNI, and valsartan is recommended by the ESC guidelines as a replacement for an ACEI to further reduce the risk of hospitalization and death in patients with HFrEF who remain symptomatic despite optimal treatment with an ACEI, a beta-blocker and an MRA. ${ }^{15} \mathrm{~A}$ case report

\section{Figure 1}

Pharmacological treatment of heart failure with reduced ejection fraction

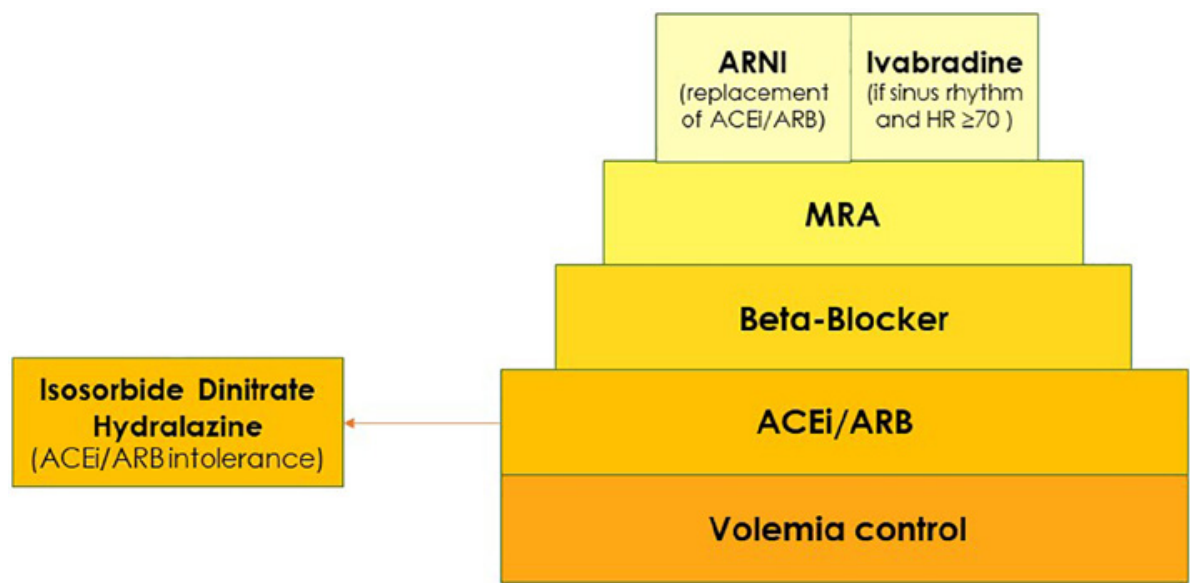

ACEI - angiotensin-converting enzime inhibitor; ARB - angiotensin-receptor blocker; ARNI - angiotensin receptor neprilysin; HR - hearth rate; MRA - mineralcorticoid receptor antagonist 
on a hemodialysis patient showed that sacubitril/valsartan was safe and improved heart failure symptoms. ${ }^{60}$ There is lack of evidence regarding the use of this drug on PD patients and more studies are needed to assess the safety and efficacy of this promising drug class.

\section{CONCLUSION}

HF and CVD remain one of the main causes of morbidity and mortality in PD patients. The importance of simple interventions such as sodium and water restriction, exercise, smoking cessation, and lifestyle modification cannot be overemphasized. Since hypervolemia is the main risk factor for HF in PD patients, prescriptions need to be tailored to achieve euvolemia. Anemia, CKD-MBD and inflammation status improvement should be considered as fundamental parts of $\mathrm{HF}$ treatment. More studies are needed to assess the efficacy and safety of the new drugs available for HF treatment in PD patients.

\section{Disclosure of potential conflicts of interest: none declared.}

\section{References}

1. Collins AJ, Foley RN, Gilbertson DT, Chen SC. United States Renal Data System public health surveillance of chronic kidney disease and end-stage renal disease. Kidney Int Suppl 2015;5(1):2-7.

2. Chiu YW, Mehrotra R. Can we reduce the cardiovascular risk in peritoneal dialysis patients? Indian J Nephrol 2010;20(2):59-67.

3. Portuguese Registry of Dialysis and Transplantation 2019. Available at http://www.bbg01.com/ cdn/rsc/spnefro/gabreg/310/ER2020_Registo.pdf. Accessed March 1, 2021.

4. Wang AY, Wang M, Lam CW, Chan IH, Lui SF, Sanderson JE. Heart failure in long-term peritoneal dialysis patients: a 4-year prospective analysis. Clin J Am Soc Nephrol 2011;6(4):805-812.

5. Wu CK, Lee JK, Wu YF, et al. Left ventricular diastolic dysfunction in peritoneal dialysis: a forgotten risk factor. Medicine (Baltimore) 2015;94(20):e819.

6. Wang $\mathrm{X}$, Shapiro Jl. Evolving concepts in the pathogenesis of uraemic cardiomyopathy. Nat Rev Nephrol 2019;15(3):159-175.

7. Krediet RT, Balafa O. Cardiovascular risk in the peritoneal dialysis patient. Nat Rev Nephrol 2010;6(8):451-460.

8. Neves M, Machado S, Rodrigues L, Borges A, Maia P, Campos M; Grupo Multicêntrico Português de Diálise Peritoneal. Cardiovascular risk in peritoneal dialysis - a Portuguese multicenter study. Nefrologia 2014;34(2):205-211.

9. Mayoor V, Prabhu BH, Santosh P, Sreedhar R, Parul K. Cardiovascular disease in peritoneal dialysis: a review, clinical queries. Nephrology 2013;2(4):152-155.

10. Terada $K$, Sakai Y, Sumi, $Y$, et al. Prognostic markers of heart failure in patients undergoing peritoneal dialysis. Ren Replace Ther 2019;5(36):995

11. Pecoits-Filho R, Barberato SH. Echocardiography in chronic kidney disease: diagnostic and prognostic implications. Nephron Clin Pract 2010;114(4):242-247.

12. Wang AY, Brimble KS, Brunier G, et al. ISPD cardiovascular and metabolic guidelines in adult peritoneal dialysis patients part II - management of various cardiovascular complications. Perit Dial Int 2015;35(4):388-396.

13. Wang AY, Lai KN. Use of cardiac biomarkers in end-stage renal disease. J Am Soc Nephrol 2008;19(9):1643-1652.

14. Paniagua $R$, Amato $D$, Mujais $S$, et al. Predictive value of brain natriuretic peptides in patients on peritoneal dialysis: results from the ADEMEX trial. Clin J Am Soc Nephrol 2008;3(2):407-415.

15. Ponikowski P, Voors AA, Anker SD, et al. ESC Guidelines for the diagnosis and treatment of acute and chronic heart failure. Eur Heart J 2016;37(27):2129-2200.

16. Zoccali C, Benedetto FA, Mallamaci F, et al. Prognostic impact of the indexation of left ventricular mass in patients undergoing dialysis. J Am Soc Nephrol. 2001;12(12):2768-2774.

17. Zoccali C, Benedetto FA, Mallamaci F, et al. Left ventricular mass monitoring in the follow-up of dialysis patients: prognostic value of left ventricular hypertrophy progression. Kidney Int 2004;65(4):1492-1498

18. Zoccali C, Benedetto FA, Mallamaci F, et al. Prognostic value of echocardiographic indicators of left ventricular systolic function in asymptomatic dialysis patients. J Am Soc Nephrol 2004;15(4):1029-1037.

19. Kennedy D, Omran E, Periyasamy SM et al. Effect of chronic renal failure on cardiac contractile function, calcium cycling, and gene expression of proteins important for calcium homeostasis in the rat. J Am Soc Nephrol 2003;14(1):90-97.

20. Segall L, Nistor I, Covic A. Heart failure in patients with chronic kidney disease: a systematic integrative review. Biomed Res Int 2014;2014:937398.

21. Pecoits-Filho R, Bucharles $\mathrm{S}$, Barberato $\mathrm{SH}$. Diastolic heart failure in dialysis patients: mechanisms, diagnostic approach, and treatment. Semin Dial 2012;25(1):35-41.
22. Herzog CA, Littrell K, Arko C, Frederick PD, Blaney M. Clinical characteristics of dialysis patients with acute myocardial infarction in the United States: a collaborative project of the United States Renal Data System and the National Registry of Myocardial Infarction. Circulation 2007;116(13):1465-1472

23. Jain $\mathrm{N}$, Hedayati SS. How should clinicians interpret cardiac troponin values in patients with ESRD? Semin Dial 2011;24(4):398-400.

24. Kalantar-Zadeh K, Abbott KC, Salahudeen AK, Kilpatrick RD, Horwich TB. Survival advantages of obesity in dialysis patients. Am J Clin Nutr 2005;81(3):543-554.

25. Johnson DW, Herzig KA, Purdie DM, et al. Is obesity a favorable prognostic factor in peritoneal dialysis patients? Perit Dial Int 2000;20(6):715-721.

26. Obi Y, Streja E, Mehrotra R, et al. Impact of obesity on modality longevity, residual kidney function, peritonitis, and survival among incident peritoneal dialysis patients. Am J Kidney Dis 2018;71(6):802$-813$.

27. Wanner C, Tonelli M, et al. KDIGO Clinical Practice Guideline for Lipid Management in CKD: summary of recommendation statements and clinical approach to the patient. Kidney Int 2014:85(6):1303-1309.

28. Jung J, Bae GH, Kang M, Kim SW, Lee DH. Statins and all-cause mortality in patients undergoing hemodialysis. J Am Heart Assoc 2020;9(5):e014840.

29. King-Morris K, Ikizler TA. Insulin resistance in patients undergoing peritoneal dialysis: can we improve it? Cardiovasc Drugs Ther 2012;26(6):441-443.

30. Shang $D$, Xie $Q, G e X$, et al. Hyperphosphatemia as an independent risk factor for coronary artery calcification progression in peritoneal dialysis patients. BMC Nephrol 2015;16:107.

31. Wang AY, Lam CW, Sanderson JE, et al. Serum 25-hydroxyvitamin D status and cardiovascular outcomes in chronic peritoneal dialysis patients: a 3-y prospective cohort study. Am J Clin Nutr 2008;87(6):1631-1638

32. Molnar MZ, Mehrotra R, Duong U, Kovesdy CP, Kalantar-Zadeh K. Association of hemoglobin and survival in peritoneal dialysis patients. Clin J Am Soc Nephrol 2011;6(8):1973-1981.

33. van der Meer P, Lipsic E, Henning RH, et al. Erythropoietin improves left ventricular function and coronary flow in an experimental model of ischemia-reperfusion injury. Eur J Heart Fail. 2004; 6(7):853-859.

34. Shahab I, Nolph KD. MIA syndrome in peritoneal dialysis: prevention and treatment. Contrib Nephrol 2006;150:135-143.

35. Mehrotra R. Long-term outcomes in automated peritoneal dialysis: similar or better than in continuous ambulatory peritoneal dialysis? Perit Dial Int 2009;29(2):111-114.

36. Rabindranath KS, Adams J, Ali TZ, Daly C, Vale L, Macleod AM. Automated vs continuous ambulatory peritoneal dialysis: a systematic review of randomized controlled trials. Nephrol Dial Transplant 2007;22(10):2991-2998

37. Srivastava S, Hildebrand S, Fan SL. Long-term follow-up of patients randomized to biocompatible or conventional peritoneal dialysis solutions show no difference in peritonitis or technique survival. Kidney Int 2011;80(9):986-991.

38. Konings CJ, Kooman JP, Schonck M, et al. Effect of icodextrin on volume status, blood pressure and echocardiographic parameters: a randomized study. Kidney Int 2003;63(4):155615-63.

39. Davies SJ, Woodrow G, Donovan K, et al. Icodextrin improves the fluid status of peritoneal dialysis patients: results of a double-blind randomized controlled trial. J Am Soc Nephrol 2003;14(9):2338-2344.

40. Bargman JM, Thorpe KE, Churchill DN; CANUSA Peritoneal Dialysis Study Group. Relative contribution of residual renal function and peritoneal clearance to adequacy of dialysis: a reanalysis of the CANUSA study. J Am Soc Nephrol 2001;12(10):2158-2162.

41. Paniagua R, Amato D, Vonesh E, et al; Mexican Nephrology Collaborative Study Group. Effects of increased peritoneal clearances on mortality rates in peritoneal dialysis: ADEMEX, a prospective, randomized, controlled trial. J Am Soc Nephrol 2002:13(5):1307-1320.

42. Wang AY, Woo J, Wang M, et al. Important differentiation of factors that predict outcome in peritoneal dialysis patients with different degrees of residual renal function. Nephrol Dial Transplant 2005 Feb;20(2):396-403.

43. Vaios V, Georgianos PI, Liakopoulos V, Agarwal R. Assessment and management of hypertension among patients on peritoneal dialysis. Clin J Am Soc Nephrol 2019;14(2):297-305.

44. Shu Y, Liu J, Zeng X, et al. The effect of overhydration on mortality and technique failure among peritoneal dialysis patients: a systematic review and meta-analysis. Blood Purif 2018:46(4):350$-358$.

45. Ng JK, Li PK. Fluid management and bioimpedance study in peritoneal dialysis. Curr Opin Nephrol Hypertens 2019; 28(1):58-64.

46. Ye $\mathrm{H}$, Zhou $\mathrm{Q}$, Fan $\mathrm{L}$, et al. The impact of peritoneal dialysis-related peritonitis on mortality in peritoneal dialysis patients. BMC Nephrol. 2017;18(1):186

47. KDIGO Clinical Practice Guideline for Anemia in Chronic Kidney Disease, Kidney Int Suppl 2012;2:279-335.

48. Mills MT. Implantable cardioverter-defibrillators in dialysis patients. BMJ Evid Based Med 2020;25:187-188.

49. Li PK, Chow KM, Wong TY, Leung CB, Szeto CC. Effects of an angiotensin converting enzyme inhibitor on residual renal function in patients receiving peritoneal dialysis. a randomized, controlled study. Ann Intern Med 2003;139(2):105-112.

50. Agarwal R, Sinha AD, Pappas MK, Abraham TN, Tegegne GG. Hypertension in hemodialysis patients treated with atenolol or lisinopril: a randomized controlled trial. Nephrol Dial Transplant 2014;29(3):672-681

51. Ito $\mathrm{Y}$, Mizuno M, Suzuki Y, et al. Long-term effects of spironolactone in peritoneal dialysis patients. J Am Soc Nephrol 2014:25(5):1094-1102.

52. Anker SD, Comin Colet J, Filippatos $\mathrm{G}$, et al. Ferric carboxymaltose in patients with heart failure and iron deficiency. N Engl J Med 2009;361(25):2436-2448.

53. Park CW, Oh YS, Shin YS, et al. Intravenous calcitriol regresses myocardial hypertrophy in hemodialysis patients with secondary hyperparathyroidism. Am J Kidney Dis 1999;33(1):73-81. 
54. Chertow GM, Block GA, Correa-Rotter R, et al. Effect of cinacalcet on cardiovascular disease in patients undergoing dialysis. N Engl J Med 2012; 367(26):2482-2494.

55. Palmer SC, Nistor I, Craig JC, et al. Cinacalcet in patients with chronic kidney disease: a cumulative meta-analysis of randomized controlled trials. PLoS Med. 2013;10(4):e1001436.

56. Liakopoulos V, Roumeliotis S, Bozikas A, Eleftheriadis T, Dounousi E. Antioxidant supplementation in renal replacement therapy patients: is there evidence? Oxid Med Cell Longev 2019;2019:9109473.

57. Feldman L, Shani M, Efrati S, et al. N-acetylcysteine improves residual renal function in peritoneal dialysis patients: a pilot study. Perit Dial Int 2011;31(5):545-550.

58. Kurpesa M, Trzos E, Wierzbowska-Drabik K, Rechciński T. Ivabradine as a heart rate-lowering agent in a patient with end-stage renal failure after heart transplantation. Kardiol Pol 2010;68(6):684 $-686$.

59. von Lueder TG, Sangaralingham SJ, Wang BH, et al. Renin-angiotensin blockade combined with natriuretic peptide system augmentation: novel therapeutic concepts to combat heart failure. Circ Heart Fail 2013;6(3):594-605.

60. Heyse A, Manhaeghe L, Mahieu E, Vanfraechem C, Van Durme F. Sacubitril/valsartan in heart failure and end-stage renal insufficiency. ESC Heart Fail 2019;6(6):1331-1333.

\section{ORCID}

Marina Reis (iD) 0000-0003-3061-3767

Catarina Almeida (iD) 0000-0001-6806-5597

Ana Marta Gomes (iD) 0000-0002-8992-5792

João Carlos Fernandes iD 0000-0003-0581-3097

\section{Correspondonce to:}

Marina Reis

R. Conceição Fernandes

4434-502 Vila Nova de Gaia, Portugal

E-mail: marina.reis9@gmail.com 